\title{
Positive solutions for singular boundary value problems involving integral conditions
}

Liang-Gen Hu*

"Correspondence:

hulianggen@yahoo.cn

Department of Mathematics,

Ningbo University, Ningbo, 315211

P.R. China

\section{Springer}

\section{Abstract}

We are interested in the following singular boundary value problem:

$$
\left\{\begin{array}{l}
u^{\prime \prime}(t)+\mu w(t) f(u(t))=0, \quad 0<t<1 \\
u(0)=0, \quad u(1)=\int_{0}^{1} u(s) d A(s)
\end{array}\right.
$$

where $\mu>0$ is a parameter and $\int_{0}^{1} u(s) d A(s)$ is the Stieltjes integral. The function $w \in C((0,1),(0,+\infty))$ and $w$ may be singular at $t=0$ and/or $t=1$,

$f \in C([0,+\infty),(0,+\infty))$ and $f_{\infty}=+\infty$. Some a priori estimates and the existence, multiplicity and nonexistence of positive solutions are obtained. Our proofs are based on the method of global continuous theorem, the lower-upper solutions methods and fixed point index theory. Furthermore, we also discuss the interval of parameter $\mu$ such that the problem has a positive solution.

Keywords: singularity; global continuous theorem; solution of boundedness; fixed point index; positive solution

\section{Introduction}

We are concerned with the second order nonlocal boundary value problem:

$$
\left\{\begin{array}{l}
u^{\prime \prime}(t)+\mu w(t) f(u(t))=0, \quad 0<t<1 \\
u(0)=0, \quad u(1)=\int_{0}^{1} u(s) d A(s)
\end{array}\right.
$$

where $\mu>0$ is a parameter and $\int_{0}^{1} u(s) d A(s)$ is a Stieltjes integral. The function $w \in$ $C((0,1),(0,+\infty))$ and $w$ may be singular at $t=0$ and/or $t=1, f \in C([0,+\infty),(0,+\infty))$ and $f_{\infty}=\lim _{u \rightarrow \infty} \frac{f(u)}{u}=+\infty$.

Integral boundary conditions and multi-point boundary conditions for differential equations come from many areas of applied mathematics and physics [1-7]. Recently, singular boundary value problems have been extensively considered in a lot of literature $[1,2$, $5,8]$, since they model many physical phenomena including gas diffusion through porous media, nonlinear diffusion generated by nonlinear sources, chemically reacting systems as well as concentration in chemical or biological problems. In all these problems, positive solutions are very meaningful.

( $) 2012 \mathrm{Hu}$; licensee Springer. This is an Open Access article distributed under the terms of the Creative Commons Attribution License (http://creativecommons.org/licenses/by/2.0), which permits unrestricted use, distribution, and reproduction in any medium, provided the original work is properly cited. 
In $[1,2]$, Webb and Infante considered the existence of positive solutions of nonlinear boundary value problem:

$$
u^{\prime \prime}(t)+\mu h(t) g(t, u(t))=0, \quad 0<t<1,
$$

where $h, g$ are nonnegative functions, subjected to the nonlocal boundary conditions

$$
u(0)=0, \quad u(1)=\alpha[u]
$$

Here $\alpha[u]$ is a linear functional on $C[0,1]$ given by

$$
\alpha[u]=\int_{0}^{1} u(s) d A(s)
$$

involving a Stieltjes integral with a signed measure, that is, $A$ has bounded variation. They dealt with many boundary conditions given in the literature in a unified way by utilizing the fixed point index theory in cones.

Recently, many researchers were interested in the global structure of positive solutions for the nonlinear boundary value problem (see, e.g., [3, 6, 7]). In 2009, Ma and An [3] considered the problem (1.1). Assume that

(A0) $A:[0,1] \rightarrow \mathbb{R}$ is nondecreasing and $A(t)$ is not a constant on $(0,1), 0 \leq \kappa<1$ with $\kappa:=\int_{0}^{1} t d A(t)$, and $\int_{0}^{1} G(t, s) d A(t) \geq 0$ for $s \in[0,1]$ (for the definition of $G(t, s)$, see (2.1) below).

(A1) $w:(0,1) \rightarrow[0,+\infty)$ is continuous and $w(t) \neq 0$ on any subinterval of $[0,1]$, and $w \Phi \in L^{1}[0,1] \cap C(0,1)$, where $\Phi(s)=s(1-s), s \in[0,1]$.

(A2) $f \in C([0,+\infty),[0,+\infty))$ and $f(s)>0$ for $s>0$.

(A3) $f_{0}=f_{\infty}=0$, where $f_{0}=\lim _{u \rightarrow 0^{+}} \frac{f(u)}{u}$ and $f_{\infty}=\lim _{u \rightarrow \infty} \frac{f(u)}{u}$.

They obtained the following main result:

Theorem 1.1 ([3, Theorem 4.1]) Assume that (AO)-(A3) hold. Then there exists a component $\mathfrak{T}$ in $\sum$ which joins $(\infty, \theta)$ to $(\infty, \infty)$, and

$$
\operatorname{Proj}_{\mathbb{R}} \mathfrak{T}=\left[\rho^{*},+\infty\right)
$$

for some $\rho^{*}>0$. Moreover, there exists $\mu^{*} \geq \rho^{*}>0$ such that (1.1) has at least two positive solutions for $\mu \in\left(\mu^{*}, \infty\right)$. Here $\mathfrak{T}$ joins $(\infty, \theta)$ to $(\infty, \infty)$ such that

$$
\lim _{\substack{(\mu, u) \in \mathfrak{T},\|u\| \leq 1 \\ \mu \rightarrow \infty}}\|u\|=0, \quad \lim _{\substack{(\mu, u) \in \mathfrak{T},\|u\|>1 \\ \mu \rightarrow \infty}}\|u\|=\infty
$$

Here, $\sum$ is the closure of the set of positive solutions of $(1.1)$ on $[0, \infty) \times X, X=\{u \in$ $\left.C^{1}[0,1]: u(0)=0, u(1)=\int_{0}^{1} u(s) d s\right\}$, and the component of a set $M$ is a maximal connected subset of $M$.

A natural problem arises: How can we consider the global structure of positive solutions for the case $f_{0}=f_{\infty}=\infty$ ?

In this paper, we first obtain the global structure of positive solutions by the use of global continuous theorem, and some a priori estimates. Applying the analysis technique, we 
construct the lower and upper solutions. Those combined with the fixed point index theory, the existence, multiplicity and nonexistence of positive solutions to (1.1) in the case $f_{0}=f_{\infty}=\infty$ are investigated. Finally, we discuss the interval of parameter $\mu$ such that the problem (1.1) has positive solutions. The proof of the method which is based on the construction of some bounds of the solution together with global continuous theorem and fixed point index is of independent interest, and is different from the other papers.

This paper is arranged as follows. We will give some hypotheses and lemmas in Section 2. In Section 3, new criteria of the existence, multiplicity and nonexistence of a positive solution are obtained. Moreover, an example is given to illustrate our result.

\section{Preliminaries and lemmas}

Let $X$ denote the Banach space $C[0,1]$ with the maximum norm

$$
\|u\|=\max _{t \in[0,1]}|u(t)| .
$$

Define $K:=\{u \in C[0,1]: u$ is concave in $[0,1]$ and $u(t) \geq 0\}$, then $K$ is a cone. Let

$$
G(t, s)= \begin{cases}(1-t) s, & 0 \leq s \leq t \leq 1 \\ (1-s) t, & 0 \leq t \leq s \leq 1\end{cases}
$$

Denote

$$
\begin{aligned}
& \kappa=\int_{0}^{1} t d A(t), \\
& G(s):=\int_{0}^{1} G(t, s) d A(t), \quad \text { for } s \in[0,1], \\
& G_{1}(t, s)=G(t, s)+\frac{t}{1-\kappa} G(s), \quad \kappa \neq 1 .
\end{aligned}
$$

Throughout this paper, we suppose that the following conditions hold:

(H0) $A:[0,1] \rightarrow \mathbb{R}$ is nondecreasing, $d A(t) \geq 0$ on $(0,1)$ and $\kappa:=\int_{0}^{1} t d A(t)$ with $0 \leq \kappa<1$.

(H1) $w \in C((0,1),(0,+\infty))$ and $w$ may be singular at $t=0$ and/or 1 , satisfying

$$
0<\int_{0}^{1} G_{1}(t, s) w(s) d s<\infty, \quad \forall t \in(0,1) .
$$

(H2) $f \in C([0, \infty),(0, \infty))(f(0)>0$ obviously holds).

(H3) $f_{\infty}=\lim _{u \rightarrow \infty} \frac{f(u)}{u}=+\infty$.

Remark 2.1 It is easy to see from (H0) that $G(s) \leq[A(1)-A(0)] G(s, s)$. Therefore, if we assume that $0<\int_{0}^{1} G(s, s) w(s) d s<\infty$, then (2.3) obviously holds.

Define an operator $T: K \rightarrow X$ as follows:

$$
T u(t)=T(\mu, u)(t):=\mu \int_{0}^{1} G_{1}(t, s) w(s) f(u(s)) d s .
$$


Assume that the conditions (H0)-(H2) hold, then it is easy to verify that $T: K \rightarrow K$ is well defined and completely continuous.

Lemma 2.1 ([9] Global continuation theorem) Let X be a Banach space and let $K$ be an order cone in $X$. Consider the equation

$$
u=T(\mu, u)
$$

where $\mu \in \mathbb{R}_{+}=[0, \infty)$ and $u \in X$. Suppose that $T: \mathbb{R}_{+} \times K \rightarrow K$ is completely continuous and $T(0, u)=\theta$ for all $u \in K$, then $\mathfrak{L}_{+}(K)$, the component of the solution set of (2.4) containing $(0,0)$ is unbounded.

\section{Remark 2.2}

(1) We note that $u$ is a positive solution of the problem (1.1) if and only if $u=T(\mu, u)$ on $K$.

(2) If $T(\mu, \theta) \neq \theta$ for $\mu \neq 0$ and $T(0, u)=\theta$ for all $u \in K$, then we get from Lemma 2.1 that there exists an unbounded continuum $\mathfrak{L}$ emanating from $(0, \theta)$ in the closure of the set of positive solutions (1.1) in $\mathbb{R}_{+} \times K$.

Lemma 2.2 ([10]) Let $X$ be a Banach space, $K$ an order cone in $X$ and $\mathcal{S}$ an open bounded set in $X$ with $\theta \in \mathcal{S}$. Suppose that $T: \overline{\mathcal{S}} \cap K \rightarrow K$ is a completely continuous operator. If $T y \neq \lambda y$, for all $y \in \partial \mathcal{S} \cap K$ and all $\lambda \geq 1$, then $i(T, \mathcal{S} \cap K, K)=1$.

\section{Main results}

Lemma 3.1 Let (HO)-(H3) hold and let $\mathcal{J}=\left[\mu_{0}, \infty\right)$ with $\mu_{0}>0$. Then there exists a constant $Q_{\mathcal{J}}>0$ such that for all $\mu \in \mathcal{J}$ and all possible positive solutions $u_{\mu}$ of (1.1), the inequality $\left\|u_{\mu}\right\|<Q_{\mathcal{J}}$ holds.

Proof Suppose on the contrary that there exist a sequence $\left\{\mu_{n}\right\} \subset \mathcal{J}$ and a sequence $\left\{u_{\mu_{n}}\right\}$ of the positive solutions of (1.1) corresponding to $\mu_{n}$ such that

$$
\left\|u_{\mu_{n}}\right\| \rightarrow \infty, \quad \text { as } n \rightarrow \infty
$$

Denote $u_{n}:=u_{\mu_{n}}$. From the concavity of $u_{n}$, it follows that

$$
u_{n}(t) \geq \frac{\left\|u_{n}\right\|}{4}>0, \quad \text { for } t \in\left[\frac{1}{4}, \frac{3}{4}\right]
$$

Choose $\eta:=\frac{10 \pi^{2}}{\mu_{0} w}$, where $w=\min _{t \in\left[\frac{1}{4}, \frac{3}{4}\right]} w(t)$. Then we find from (H3) that there exists a constant $R>0$ such that

$$
f(u)>\eta u, \quad \text { for all } u>R \text {. }
$$

Since $\lim _{n \rightarrow \infty}\left\|u_{n}\right\|=\infty$, we get

$$
\left\|u_{N}\right\|>4 R, \quad \text { for enough large } N \text {. }
$$


This, together with (3.1) and (3.2), implies

$$
f\left(u_{N}(t)\right)>\eta u_{N}(t), \quad \text { for } t \in\left(\frac{1}{4}, \frac{3}{4}\right)
$$

Put $\psi(t):=-\cos 2 \pi t$, for $t \in\left[\frac{1}{4}, \frac{3}{4}\right]$. Hence, we know from (3.3) that

$$
\begin{aligned}
\mu_{N} \int_{\frac{1}{4}}^{\frac{3}{4}} w(t) f\left(u_{N}(t)\right) \psi(t) d t & \geq \eta \mu_{N} \int_{\frac{1}{4}}^{\frac{3}{4}} w(t) u_{N}(t) \psi(t) d t \\
& \geq \eta w \mu_{N} \int_{\frac{1}{4}}^{\frac{3}{4}} u_{N}(t) \psi(t) d t
\end{aligned}
$$

On the other hand, multiplying (1.1) by $\psi$ and integrating by parts, we obtain that

$$
\begin{aligned}
\mu_{N} \int_{\frac{1}{4}}^{\frac{3}{4}} w(t) f\left(u_{N}(t)\right) \psi(t) d t & =-\int_{\frac{1}{4}}^{\frac{3}{4}} u_{N}^{\prime \prime}(t) \psi(t) d t \\
& =-\left.u_{N}^{\prime}(t) \psi(t)\right|_{\frac{1}{4}} ^{\frac{3}{4}}+\int_{\frac{1}{4}}^{\frac{3}{4}} u_{N}^{\prime}(t) \psi^{\prime}(t) d t \\
& =-\int_{\frac{1}{4}}^{\frac{3}{4}} u_{N}(t) \psi^{\prime \prime}(t) d t+\left.u_{N}(t) \psi^{\prime}(t)\right|_{\frac{1}{4}} ^{\frac{3}{4}} \\
& \leq 4 \pi^{2} \int_{\frac{1}{4}}^{\frac{3}{4}} u_{N}(t) \psi(t) d t
\end{aligned}
$$

leads to

$$
\left.\eta w \mu_{N} \int_{\frac{1}{4}}^{\frac{3}{4}} u_{N}(t) \psi(t) d t \leq 4 \pi^{2} \int_{\frac{1}{4}}^{\frac{3}{4}} u_{N}(t) \psi(t)\right] d t
$$

i.e.,

$$
\frac{10 \pi^{2}}{\mu_{0} w}=\eta \leq \frac{4 \pi^{2}}{\mu_{N} w}<\frac{10 \pi^{2}}{\mu_{0} w}
$$

This is a contradiction.

Lemma 3.2 Assume that the hypotheses (HO)-(H3) hold. Then there exists $\rho>0$ such that if the problem (1.1) has a positive solution for parameter $\mu$, then $\mu \leq \rho$.

Proof Let $u$ be a positive solution of (1.1) corresponding to $\mu$. From the hypotheses (H2) and (H3), it follows that there exists $\varrho>0$ such that $f(u) \geq \varrho u$ for all $u>0$. Consequently, we have

$$
\mu \varrho w(t) u(t) \leq \mu w(t) f(u(t))=-u^{\prime \prime}(t), \quad t \in(0,1) .
$$


Let $\lambda_{1}$ be the first eigenvalue of

$$
\left\{\begin{array}{l}
\varphi^{\prime \prime}(t)+\lambda w(t) \varphi(t)=0, \quad t \in(0,1) \\
\varphi(0)=\varphi(1)=0
\end{array}\right.
$$

and let $\varphi_{1}$ be the positive eigenfunction corresponding to $\lambda_{1}$ (see [8]). It is easy to see that $\varphi_{1}^{\prime}(1) \leq 0$. Multiplying (3.4) by $\varphi_{1}$ and integrating by parts, we get that

$$
\begin{aligned}
\mu \varrho \int_{0}^{1} w(t) u(t) \varphi_{1}(t) d t & \leq-\int_{0}^{1} u^{\prime \prime}(t) \varphi_{1}(t) d t \\
& =-\left.\varphi_{1}(t) u^{\prime}(t)\right|_{0} ^{1}+\int_{0}^{1} u^{\prime}(t) \varphi_{1}^{\prime}(t) d t \\
& =u(1) \varphi_{1}^{\prime}(1)-u(0) \varphi_{1}^{\prime}(0)-\int_{0}^{1} u(t) \varphi_{1}^{\prime \prime}(t) d t \\
& \leq-\int_{0}^{1} u(t) \varphi_{1}^{\prime \prime}(t) d t \\
& =\lambda_{1} \int_{0}^{1} w(t) u(t) \varphi_{1}(t) d t
\end{aligned}
$$

implies

$$
\mu \leq \lambda_{1} \varrho^{-1} \triangleq \rho .
$$

This completes the proof.

Lemma 3.3 Assume that (HO)-(H3) hold. Then we have

$$
\lim _{\substack{\left(\mu, u_{\mu}\right) \in \mathfrak{L} \\\left\|u_{\mu}\right\| \geq 1, \mu \rightarrow 0}}\left\|u_{\mu}\right\|=\infty
$$

Proof Suppose this fails, that is, there exists $\left\{\left(\mu, u_{\mu}\right)\right\} \subset \mathfrak{L}$ such that

$$
\mu \rightarrow 0 \text { and } \quad 1 \leq\left\|u_{\mu}\right\| \leq M_{0}
$$

where $M_{0}$ is a positive constant. Since $\left(\mu, u_{\mu}\right) \in \mathfrak{L}$, we get that for all $t \in[0,1]$

$$
\begin{aligned}
u_{\mu}(t) & =\mu \int_{0}^{1} G_{1}(t, s) w(s) f\left(u_{\mu}(s)\right) d s \\
& =\left(\mu \int_{0}^{1} G_{1}(t, s) w(s) \frac{f\left(u_{\mu}(s)\right)}{\left\|u_{\mu}\right\|} d s\right)\left\|u_{\mu}\right\| \\
& \leq \mu\left(M_{1} \max _{t \in[0,1]} \int_{0}^{1} G_{1}(t, s) w(s) d s\right)\left\|u_{\mu}\right\|,
\end{aligned}
$$

where

$$
M_{1}=\max \left\{\frac{f\left(u_{\mu}(t)\right)}{\left\|u_{\mu}\right\|}: 1 \leq\left\|u_{\mu}\right\| \leq M_{0}, t \in[0,1]\right\} \in(0, \infty) .
$$


Hence, we find that

$$
\mu \geq\left\{M_{1} \max _{t \in[0,1]} \int_{0}^{1} G_{1}(t, s) w(s) d s\right\}^{-1}>0 .
$$

Thus, it implies that $\mu \nrightarrow 0$. This is contradiction.

Theorem 3.1 Assume that the conditions (HO)-(H3) hold and $\int_{0}^{1} d A(t)<1$. Then there exists a constant $\mu_{1}>0$ such that the problem (1.1) has at least two positive solutions for $0<\mu<\mu_{1}$, and at least one positive solution for $\mu=\mu_{1}$, and no positive solution for $\mu>\mu_{1}$.

Proof Define

$$
\mu_{1}:=\sup \left\{\mu^{*}>0: \text { for all } \mu \in\left(0, \mu^{*}\right)\right.
$$

there exist at least two positive solutions of $(1.1)\}$.

From Lemma 2.1 and Remark 2.2, we can find that there exists an unbounded continuum $\mathfrak{L}$ emanating from $(0, \theta)$ in the closure of the set of positive solutions in $\mathbb{R}_{+} \times K$ and $T(0, u)=\theta$ for all $u \in K$. Meanwhile, Lemma 3.1 and Lemma 3.3 respectively imply that $u_{\mu}$ is bounded $(\mu>0, \mu \nrightarrow 0)$ and unbounded $\left(\mu>0, \mu \rightarrow 0\right.$ and $\left.\left\|u_{\mu}\right\| \geq 1\right)$. Therefore, we conclude that the set of (3.5) is nonempty. Those combined with Lemma 3.2 follows that $\mu_{1}>0$ is well defined and $\mu_{1} \in(0, \rho]$. From the definition of $\mu_{1}$, it is easy to see that the problem (1.1) has at least two positive solutions for $\mu \in\left(0, \mu_{1}\right)$. Again, since the continuum is a compact connected set and $T$ is a completely continuous operator, the problem (1.1) has at least one positive solution at $\mu=\mu_{1}$.

Next, we only show that the problem (1.1) has no positive solution for any $\mu>\mu_{1}$. Suppose on the contrary that there exists some $\mu_{2}\left(>\mu_{1}\right)$ such that the problem (1.1) has a positive solution $u_{2}$ corresponding to $\mu_{2}$. Then we will prove that the problem (1.1) has at least two positive solutions for any $\mu \in\left[\mu_{1}, \mu_{2}\right)$ which contradicts the definition of (3.5).

For the sake of obtaining the contradiction, we divide the proof into four steps.

Step 1. Constructing a modified boundary value problem.

Choose arbitrarily a constant $\mu \in\left[\mu_{1}, \mu_{2}\right)$. Since $f$ is uniformly continuous on $\left[0,\left\|u_{2}\right\|+\right.$ $1]$, there exists a constant $\sigma>0$ such that

$$
f(u+\sigma)<f(u)+\epsilon, \quad \text { for } u \in\left[0,\left\|u_{2}\right\|+1\right]
$$

where

$$
\epsilon:=\frac{\left(\mu_{2}-\mu\right) \min _{u \in\left[0,\left\|u_{2}\right\|+1\right]} f(u)}{2 \mu}>0 .
$$

Denote $\zeta(t):=u_{2}(t)+\sigma$, for $t \in[0,1]$. Then we claim that

$$
\zeta^{\prime \prime}(t)+\mu w(t) f(\zeta(t))<0, \quad t \in(0,1),
$$

and

$$
\zeta(0)=\sigma, \quad \zeta(1)=\int_{0}^{1} u_{2}(s) d A(s)+\sigma .
$$


Indeed, using (3.6), we have

$$
\begin{aligned}
\zeta^{\prime \prime}(t)+\mu w(t) f(\zeta(t)) & =u_{2}^{\prime \prime}(t)+\mu w(t) f\left(u_{2}(t)+\sigma\right) \\
& =-\mu_{2} w(t) f\left(u_{2}(t)\right)+\mu w(t) f\left(u_{2}(t)+\sigma\right) \\
& <w(t)\left[-\mu_{2} f\left(u_{2}(t)\right)+\mu f\left(u_{2}(t)\right)+\mu \epsilon\right] \\
& \leq-\frac{\mu_{2}-\mu}{2} \times w(t) f\left(u_{2}(t)\right) \\
& <0 .
\end{aligned}
$$

Define a set $\mathcal{S}:=\{u \in C[0,1] \mid 0<u(t)<\zeta(t), t \in(0,1)\}$. Then the set $\mathcal{S}$ is bounded and open in $C[0,1]$. Now, we construct the modified second-order boundary value problem:

$$
\left\{\begin{array}{l}
u^{\prime \prime}(t)+\mu w(t) f(\xi(u(t)))=0, \quad t \in(0,1) \\
u(0)=0, \quad u(1)=\int_{0}^{1} u(s) d A(s)
\end{array}\right.
$$

where $\xi: \mathbb{R} \rightarrow \mathbb{R}_{+}$is defined by

$$
\xi(u(t))= \begin{cases}\zeta(t), & \text { if } u(t)>\zeta(t) \\ u(t), & \text { if } 0 \leq u(t) \leq \zeta(t) \\ 0, & \text { if } u(t)<0\end{cases}
$$

Step 2. We will show that if $u$ is a positive solution of (3.9), then $u \in \mathcal{S} \cap K$.

Let $u$ be a positive solution of (3.9), then we claim that

$$
u \in \mathcal{S} \cap K .
$$

Suppose this fails, that is, $u \notin \mathcal{S} \cap K$. Clearly, we only show that $u(t) \nless \zeta(t)$, for $t \in(0,1]$. Comparing the boundary conditions (3.8) and (3.9), the only following three cases need to be considered. Case I. There exists $t_{1} \in(0,1)$ such that $u\left(t_{1}\right)=\zeta\left(t_{1}\right)$ and $0<u(t)<\zeta(t)$, for $t \in\left(t_{1}-\sigma_{1}, t_{1}\right) \cup\left(t_{1}, t_{1}+\sigma_{1}\right)$ and some $\sigma_{1}>0$; Case II. There exists $t_{2} \in(0,1]$ such that $u\left(t_{2}\right)=\zeta\left(t_{2}\right)$ and $u(t) \geq \zeta(t)$ for $t \in\left(t_{2}, 1\right]$. Case III. There exists $\left[t_{3}, t_{4}\right] \subset(0,1]$ such that $u(t) \geq \zeta(t), t \in\left[t_{3}, t_{4}\right], u\left(t_{3}\right)=\zeta\left(t_{3}\right)$ and $u\left(t_{4}\right)=\zeta\left(t_{4}\right)$. See the three Figures 1,2 and 3 .

Case I. From (3.7), it implies that there exists a constant $\epsilon_{1}>0$ such that

$$
\max _{t \in\left[t_{1}-\sigma_{1}, t_{1}+\sigma_{1}\right]}\left\{\zeta^{\prime \prime}(t)+\mu w(t) f(\zeta(t))\right\}=-\epsilon_{1}<0 .
$$

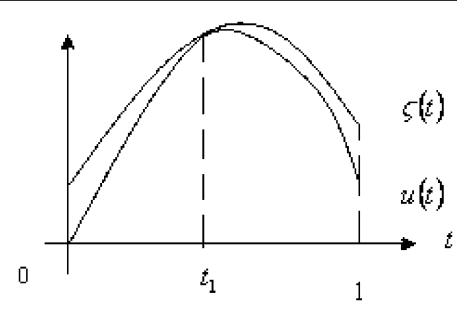

Figure 1 Case I. 


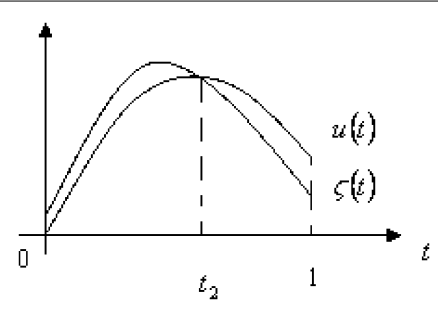

Figure 2 Case II.

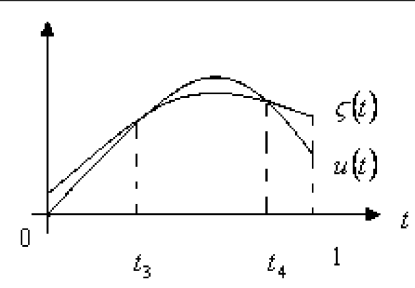

Figure 3 Case III.

Since $f$ is uniformly continuous on $\left[0,\left\|u_{2}\right\|+1\right]$, there exists a $\sigma_{1}^{\prime}>0$ such that if $u, v \in$ $[0,\|\zeta\|]$ and $|u-v|<\sigma_{1}^{\prime}$, then we get

$$
|f(u)-f(v)|<\epsilon_{1} W^{-1},
$$

where $W:=\mu \max _{t \in\left[t_{1}-\sigma_{1}, t_{1}+\sigma_{1}\right]} w(t)>0$. From the assumption of Case I, it follows that there exists a subinterval $[r, s] \subset\left(t_{1}-\sigma_{1}, t_{1}+\sigma_{1}\right)$ with $t_{1} \in(r, s)$ such that

$$
-\sigma_{1}^{\prime}<u(t)-\zeta(t) \leq 0, \quad \text { for } t \in[r, s]
$$

and

$$
(u-\zeta)^{\prime}(r)>0 \quad \text { and } \quad(u-\zeta)^{\prime}(s)<0 .
$$

This together with (3.11) and (3.12) leads to

$$
\begin{aligned}
0 & >(u-\zeta)^{\prime}(s)-(u-\zeta)^{\prime}(r)=\int_{r}^{s}\left[u^{\prime \prime}(t)-\zeta^{\prime \prime}(t)\right] d t \\
& =-\int_{r}^{s}\left[\mu w(t) f(u(t))+\zeta^{\prime \prime}(t)\right] d t \\
& =-\int_{r}^{s}\left[\mu w(t)(f(u(t))-f(\zeta(t)))+\zeta^{\prime \prime}(t)+\mu w(t) f(\zeta(t))\right] d t \\
& \geq-\int_{r}^{s}\left[\mu w(t) \epsilon_{1} W^{-1}-\epsilon_{1}\right] d t \\
& =\varepsilon_{1} \int_{r}^{s}\left[1-\frac{\mu w(t)}{W}\right] d t \geq 0 .
\end{aligned}
$$

This is a contradiction. 
Case II. Let $x(t):=u(t)-\zeta(t)$, for $t \in[0,1]$. Then we have that for $t \in\left(t_{2}, 1\right]$

$$
\begin{aligned}
& x\left(t_{2}\right)=0, \quad x(t) \geq 0 \\
& x^{\prime \prime}(t)=(u(t)-\zeta(t))^{\prime \prime}=-\mu w(t) f(\zeta(t))-\zeta^{\prime \prime}(t)>0 .
\end{aligned}
$$

Obviously, we have $x^{\prime}\left(t_{2}\right) \geq 0$. From (3.13), it implies that $x^{\prime}(t)>0$, for any $t \in\left(t_{2}, 1\right]$. Hence, the function $x(t)$ is strictly increasing in $\left[t_{2}, 1\right]$. From $(3.8)$ and the boundary condition (3.9), we find

$$
\begin{aligned}
x(1) & =u(1)-\zeta(1)=\int_{0}^{1} u(s) d A(s)-\int_{0}^{1} u_{2}(s) d A(s)-\sigma \\
& =\int_{0}^{1}(u(s)-\zeta(s)) d A(s)+\sigma\left(\int_{0}^{1} d A(s)-1\right) \\
& =\int_{0}^{1} x(s) d A(s)-\sigma\left(1-\int_{0}^{1} d A(s)\right) \\
& <x(1) .
\end{aligned}
$$

We obtain a contradiction. In particular, if $t_{2}=1$, then we obtain

$$
x(1)=u(1)-\zeta(1)=\int_{0}^{1} x(s) d A(s)+\sigma\left(\int_{0}^{1} d A(s)-1\right)<0 .
$$

This contradicts (3.13).

Case III. Since $\xi(u(t))=\zeta(t)$, for $t \in\left(t_{3}, t_{4}\right)$, we have that $(u(t)-\zeta(t))^{\prime \prime}>0$, for $t \in\left(t_{3}, t_{4}\right)$. Again since $(u-\zeta)\left(t_{3}\right)=(u-\zeta)\left(t_{4}\right)=0$, we know from the maximum principle that $u(t)<$ $\zeta(t)$, for $t \in\left(t_{3}, t_{4}\right)$. This contradicts the assumption of Case III.

Therefore, we conclude that the claim (3.10) holds.

Step 3. $i\left(\widetilde{T}_{\mu}, \mathcal{S} \cap K, K\right)=1$ (the definition of $\widetilde{T}_{\mu}$ see below).

Using (2.2), we define an operator $\widetilde{T}_{\mu}: K \rightarrow X$ by

$$
\widetilde{T}_{\mu}(u)(t)=\mu \int_{0}^{1} G_{1}(t, s) w(s) f(\xi(u(s))) d s, \quad t \in[0,1] .
$$

Then $\widetilde{T}_{\mu}: K \rightarrow K$ is completely continuous and $u$ is a positive solution of (3.9) if and only if $u=\widetilde{T}_{\mu}(u)$ on $K$. From the definition of $\xi(u(t))$, it implies that there exists $R_{1}>0$ such that $\left\|\widetilde{T}_{\mu} u\right\|<R_{1}$, for all $u \in K$. Consequently, we get from Lemma 2.2 that

$$
i\left(\widetilde{T}_{\mu}, B_{R_{1}} \cap K, K\right)=1
$$

where $B_{R_{1}}=\left\{u \in X:\|u\|<R_{1}\right\}$. Applying the conclusion of Step 2 and the excision property of fixed point index, we find that

$$
i\left(\widetilde{T}_{\mu}, \mathcal{S} \cap K, K\right)=i\left(\widetilde{T}_{\mu}, B_{R_{1}} \cap K, K\right)=1 .
$$

Step 4. We conclude that the problem (1.1) has at least two positive solutions corresponding to $\mu$. 
Since the problem (1.1) is equivalent to the problem (3.9) on $\mathcal{S} \cap K$, we get that the problem (1.1) has a positive solution in $\mathcal{S} \cap K$. Without loss of generality, we may suppose that $T$ has no fixed point on $\partial \mathcal{S} \cap K$ (otherwise the proof is completed). Then $i(T, \mathcal{S} \cap K, K)$ is well defined and (3.15) implies

$$
i(T, \mathcal{S} \cap K, K)=1
$$

On the other hand, from Lemma 3.2, we choose $\mu_{*}>\rho$ such that the problem (1.1) has no positive solution in $K$. By a priori estimate in $\mathcal{J}=\left[\mu, \mu_{*}\right]$, there exists $R_{2}\left(>R_{1}\right)>0$ such that for all possible positive solutions $u_{\lambda}$ of (1.1) with $\lambda \in\left[\mu, \mu_{*}\right]$, we know that $\left\|u_{\lambda}\right\|<R_{2}$. Define $\mathfrak{G}:[0,1] \times\left(\bar{B}_{R_{2}} \cap K\right) \rightarrow K$ by

$$
\mathfrak{G}(v, u)=T(v \mu+(1-v) \mu *, u)
$$

Then it is easy to verify that $\mathfrak{G}$ is completely continuous on $[0,1] \times K$ and $\mathfrak{G}(v, u) \neq u$ for all $(v, u) \in[0,1] \times\left(\partial B_{R_{2}} \cap K\right)$. From the property of homotopy invariance, it follows that

$$
i\left(T, B_{R_{2}} \cap K, K\right)=i\left(T(\mu, \cdot), B_{R_{2}} \cap K, K\right)=i\left(T\left(\mu_{*}, \cdot\right), B_{R_{2}} \cap K, K\right)=0 .
$$

Hence, by the additivity property and (3.16), we have

$$
i\left(T,\left(B_{R_{2}} \backslash \overline{\mathcal{S}}\right) \cap K, K\right)=-1 .
$$

Then we conclude that the problem (1.1) has at least two positive solutions corresponding to $\mu$.

\section{Remark 3.1}

(i) From the hypotheses (H2) and (H3), it implies that there exists $L>0$ such that

$$
\mathcal{D}=\frac{f(L)}{L}=\min _{u>0} \frac{f(u)}{u} .
$$

Let $f$ attain its maximum at the point $L^{*}$ of $[0, L]$. If $\int_{0}^{1} G_{1}(t, s) w(s) d s<\infty$, then adopting the similar method as in [11, Theorem 1], we get that for $0<\mu<\left(\frac{f\left(L^{*}\right)}{L} \int_{0}^{1} G_{1}(t, s) w(s) d s\right)^{-1}$, the problem (1.1) has at least two positive solutions $u_{1}(t)$ and $u_{2}(t)$ such that $0<\left\|u_{1}\right\|<L<\left\|u_{2}\right\|$ by the use of compression of conical shells in [12, Corollary 20.1]. Consequently, we know that $\mu_{1} \geq\left(\frac{f\left(L^{\prime \prime}\right)}{L} \int_{0}^{1} G_{1}(t, s) w(s) d s\right)^{-1}$.

(ii) If $u$ is a positive solution of the equation (1.1) corresponding to $\mu$, then we have

$$
\|u\|=\max _{t \in[0,1]}|u(t)|=\mu \max _{t \in[0,1]} \int_{0}^{1} G_{1}(t, s) w(s) f(u(s)) d s
$$

i.e., from (3.17),

$$
1=\mu \max _{t \in[0,1]} \int_{0}^{1} G_{1}(t, s) w(s) \frac{f(u(s))}{\|u\|} d s \geq \mu \mathcal{D} \max _{t \in[0,1]} \int_{0}^{1} G_{1}(t, s) w(s) d s .
$$

Therefore, we get that $\mu^{*} \leq\left(\mathcal{D} \max _{t \in[0,1]} \int_{0}^{1} G_{1}(t, s) w(s) d s\right)^{-1}$. 
Corollary 3.1 Assume that (H1)-(H3) hold. Consider the following m-point boundary value problem

$$
\left\{\begin{array}{l}
u^{\prime \prime}(t)+\mu w(t) f(u(t))=0, \quad 0<t<1, \\
u(0)=0, \quad u(1)=\sum_{i=1}^{m-2} \alpha_{i} u\left(\eta_{i}\right),
\end{array}\right.
$$

where $\mu$ is a positive parameter, $\alpha_{i} \in[0, \infty), i=1,2, \ldots, m-2,0<\eta_{1}<\cdots<\eta_{m-2}<1$ and $0 \leq \sum_{i=1}^{m-2} \alpha_{i} \eta_{i}<1$. Then there exists a constant $\bar{\mu}>0$ such that the problem (3.18) has at least two positive solutions for $0<\mu<\bar{\mu}$, and at least one positive solution for $\mu=\bar{\mu}$, and no positive solution for $\mu>\bar{\mu}$.

Proof In the boundary condition of (1.1), if we let

$$
A(s)=\sum_{i=1}^{m-2} \alpha_{i} \chi\left(s-\eta_{i}\right)
$$

where $\chi(s)$ is the characteristic function on $[0, \infty)$, i.e.,

$$
\chi(s)= \begin{cases}1, & \text { if } s \geq 0, \\ 0, & \text { if } s<0 .\end{cases}
$$

Then the boundary condition of (1.1) reduces to the $m$-point boundary condition of (3.18). Applying the method of Theorem 3.1, we get the conclusion.

Example 3.1 We consider the following singular boundary value problem:

$$
\left\{\begin{array}{l}
u^{\prime \prime}(t)+\mu \frac{1}{\sqrt[3]{t}}\left(2+\sin u(t)+e^{u(t)}\right)=0, \quad t \in(0,1), \\
u(0)=0, \quad u(1)=\int_{0}^{1} u(s) d A(s),
\end{array}\right.
$$

where $A(s)=s^{2}(2-s)$.

\section{Computing yields}

$$
\begin{aligned}
& \begin{array}{l}
\kappa=\int_{0}^{1} t d A(t)=\int_{0}^{1} t d\left(2 t^{2}-t^{3}\right)=\frac{7}{12}, \\
G(s)=\int_{0}^{1} G(t, s) d A(t) \\
\quad=\int_{0}^{s}(1-s) t\left(4 t-3 t^{2}\right) d t+\int_{s}^{1}(1-t)\left(4 t-3 t^{2}\right) s d t \\
\quad=\frac{5}{12} s-\frac{2}{3} s^{3}+\frac{1}{4} s^{4},
\end{array} \\
& G_{1}(t, s)=G(t, s)+\frac{t}{7}\left(5 s-8 s^{3}+3 s^{4}\right) .
\end{aligned}
$$


We find that for any $t \in(0,1]$,

$$
\begin{aligned}
0 & <\int_{0}^{1} G_{1}(t, s) w(s) d s \\
& =\int_{0}^{1} \frac{G(t, s)}{\sqrt[3]{s}} d s+\frac{t}{7} \int_{0}^{1} s^{\frac{2}{3}}\left(5-8 s^{2}+3 s^{3}\right) d s \\
& =t\left(\frac{2,988}{2,695}-\frac{9}{10} t^{2}\right)<\infty .
\end{aligned}
$$

Thus, it implies that (2.3) holds. It is easy to verify that the conditions (H2) and (H3) hold. Therefore, by Theorem 3.1, we obtain that there exists a constant $\mu_{1}>0$ such that the problem (3.19) has at least two positive solutions for $0<\mu<\mu_{1}$, and at least one positive solution for $\mu=\mu_{1}$, and no positive solution for $\mu>\mu_{1}$.

\section{Competing interests}

The author declares that they have no competing interests.

\section{Author's contributions}

The author typed, read and approved the final manuscript.

\section{Acknowledgement}

The author would like to thank the anonymous referees very much for helpful comments and suggestions which led to the improvement of presentation and quality of the work. The work was supported partly by NSCF of Tianyuan Youth Foundation (No. 11126125), K. C. Wong Magna Fund of Ningbo University, Subject Foundation of Ningbo University (No. xkl11044) and Hulan's Excellent Doctor Foundation of Ningbo University.

\section{Received: 20 December 2011 Accepted: 1 June 2012 Published: 5 July 2012}

\section{References}

1. Webb, JRL, Infante, G: Positive solutions of nonlocal boundary value problems: a unified approach. J. Lond. Math. Soc. 74, 673-693 (2006)

2. Webb, JRL, Infante, G: Positive solutions of nonlocal boundary value problems involving integral conditions. Nonlinear Differ. Equ. Appl. 15, 45-67 (2008)

3. $\mathrm{Ma}, \mathrm{RY}, \mathrm{An}, \mathrm{YL}$ : Global structure of positive solutions for nonlocal boundary value problems involving integral conditions. Nonlinear Anal. TMA 71, 4364-4376 (2009)

4. Anderson, DR, Smyrlis, G: Solvability for a third-order three-point boundary value problem on time scales. Math. Comput. Model. 49, 1994-2001 (2009)

5. Lan, KQ, Webb, JRL: Positive solutions of semilinear differential equations with singularities. J. Differ. Equ. 148, 407-421 (1998)

6. Li, HY, Sun, JX: Positive solutions of superlinear semipositone nonlinear boundary value problems. Comput. Math. Appl. 61, 2806-2815 (2011)

7. Rynne, B: Spectral properties and nodal solutions for second-order, $m$-point, boundary value problems. Nonlinear Anal. TMA 67, 3318-3327 (2007)

8. Asakawa, H: On nonresonant singular two-point boundary value problems. Nonlinear Anal. 47, 4849-4860 (2001)

9. Zeidler, E: Nonlinear Functional Analysis and Its Applications, I. Fixed-Point Theorems. Springer, New York (1986)

10. Guo, DJ, Lakshmikantham, V: Nonlinear Problems in Abstract Cones. Academic Press, New York (1988)

11. Sánchez, J: Multiple positive solutions of singular eigenvalue type problems involving the one-dimensional p-Laplacian. J. Math. Anal. Appl. 292, 401-414 (2004)

12. Deimling, K: Nonlinear Functional Analysis. Springer, Berlin (1985) 